\title{
Quantitative Analysis of the Process and Propagation of Cortical Granule Breakdown in Sea Urchin Eggs
}

\author{
Tatsuma Mohri and Yukihisa Hamaguchi \\ Biological Laboratory, Tokyo Institute of Technology, O-okayama, Meguro-ku, Tokyo 152, Japan
}

Key words: Ca-EGTA buffer/cortical granule breakdown/fertilization $/ \mathrm{IP}_{3} /$ sea urchin egg/video microscope

\begin{abstract}
Cortical granule breakdown in sea urchin eggs has been investigated with a video microscope system using Nomarski differential interference contrast optics, when induced by fertilization, microinjecting inositol 1, 4, 5-trisphosphate ( $\left.{ }^{2} P_{3}\right)$ or Ca-EGTA buffer solution into the egg, or perfusing a medium containing $1 \mathrm{mM}$ $\mathrm{Ca}^{2+}$ to isolated cortices. The cortical granule increased up to 1.2 times in diameter and broke down within 40 msec. These values were almost constant among the three methods used to induce cortical granule breakdown. Upon fertilization, the cortical granule breakdown propagated over the egg surface at a speed of $3.3 \mu \mathrm{m} / \mathrm{sec}$ in Clypeaster japonicus eggs, which indicates that cortical granule breakdown propagated through the $3.3-\mu \mathrm{m}$ wide egg surface within 1 sec. In such a small area of the egg surface, however, it took much more than 1 sec for all cortical granules to break down because the maximal rate of breakdown was $7.6 \% / \mathrm{sec}$; that is, it took $9 \mathrm{sec}$ and 18 sec for $50 \%$ and $90 \%$, respectively, of cortical granules to break down. Moreover, the rate did not simply decrease with time, and a shoulder was found during the reducing phase, which suggests that cortical granules are divided into fast and slow breakdown groups according to the responsiveness to the breakdown stimulus. The cortical granule breakdown induced by microinjecting the Ca-EGTA buffer and IP ${ }_{3}$ solutions propagated at $68 \mu \mathrm{m} / \mathrm{sec}$ and $35 \mu \mathrm{m} / \mathrm{sec}$, respectively. The stimulus for cortical granule breakdown is discussed concerning the transient intracellular $\mathrm{Ca}^{2+}$ increase.
\end{abstract}

Upon fertilization of the echinoderm egg, it is well known that the formation of the fertilization envelope is caused by cortical granule breakdown (CGB) $(7,11$, $13,19,23$ ). Following the attachment of a spermatozoon at the egg surface, the inclusion of cortical granules (CGs) protrudes into the space between the plasma membrane and the vitelline envelope, and it causes the elevation of the fertilization envelope, forming the perivitelline space $(7,19)$. CGB starts at a site near sperm incorporation upon fertilization, and in the case of the microinjection of $\mathrm{Ca}^{2+}$ and inositol 1, 4, 5-trisphosphate $\left(\mathrm{IP}_{3}\right)$, it starts at the vicinity of the injection site. Subsequently, it spreads over the entire surface of the egg in a wavelike manner $(11,12,24)$. According to precise observation, however, adjacent CGs do not simultaneously break down, but seem to break down at random $(4,12)$. In the present study, the process of CGB was quantitatively analyzed by video microscopy.

Abbreviations: ASW, artificial sea water; $\mathrm{CG}$, cortical granule; CGB, cortical granule breakdown; EGTA, ethyleneglycol bis $(\beta-$ aminoethylether) tetraacetic acid; $\mathrm{IP}_{3}$, inositol 1, 4, 5-trisphosphate;

\section{MATERIALS AND METHODS}

Materials. Eggs were obtained from the sea urchins, Temnopleurus hardwicki and Hemicentrotus pulcherrimus and the sand dollars, Scaphechinus mirabilis and Clypeaster japonicus by intracoelomic injection of artificial sea water (ASW; Jamarin U: Jamarin Laboratory, Osaka) containing 1 $\mathrm{mM}$ acetylcholine. The eggs were washed three times with ASW and kept at $15^{\circ} \mathrm{C}$ before use. Sperm were kept "dry" at $4^{\circ} \mathrm{C}$.

Induction of CGB. All experiments were carried out at $20 \pm 1^{\circ} \mathrm{C}$ in the case of $S$. mirabilis and $H$. pulcherrimus, and at $25 \pm 1{ }^{\circ} \mathrm{C}$ in the case of T. hardwicki and C. japonicus. CGB was induced by insemination and microinjection of $100 \mathrm{mM}$ Ca-EGTA buffer $\left(6 \mu \mathrm{M}\right.$ at the concentration of $\left.\mathrm{Ca}^{2+}\right)$ and $\mathrm{IP}_{3}$ solution $(20 \mu \mathrm{M})$ as reported previously (12). Eggs in a trough made by a coverslip were inseminated by adding a drop of sperm suspension which was obtained by diluting dry sperm with ASW up to 500-1000 times. Microinjection was carried out in the same way, as reported previously by Mohri and Hamaguchi (12). The volume of injected solution per egg was 6-7 pl corresponding to $1 \%$ of the egg volume. Cortices of the eggs were isolated and perfused with a solution containing 1 $\mathrm{mM} \mathrm{Ca}{ }^{2+}$ in order to induce CGB by the method of Sasaki 
and Epel (18).

Observation and analysis. $\mathrm{CGB}$ was observed with a Nomarski differential interference microscope (Optiphot; Nikon Corp., Tokyo) using a $100 \times / 1.25$ Nikon DIC objective and recorded with a video system at a speed of 60 fields/ $\sec (12)$ or at a speed of 200 fields/sec using a high-speed video camera, MHS-200 (Nac Incorporated, Tokyo).

The periods in msec required for the breakdown of a single $C G$ were determined to be 5 times the number of fields, from the video field where any morphological change in CG was detected, to the video field where CG had disappeared because one video field at $200 \mathrm{fields} / \mathrm{sec}$ is equal to $5 \mathrm{msec}$.

Only a part of the egg surface could be observed with the microscope because the egg was spherical. Therefore, the egg was compressed somewhat, the focus was adjusted to the top surface of the compressed egg and the observation area was a rectangle $(51 \times 37 \mu \mathrm{m})$ which was a full frame of the image on a video monitor (see Fig. 2a). Positions of all CGs in the image were traced on transparent plastic sheets using a fine-point marker pen and all times of their breakdown in minutes and seconds after insemination or microinjection were noted on the sheet. The breakdown time of individual CGs was measured by reviewing both the recorded images of $\mathrm{CG}$ and the time superimposed in the image field in slow motion or still shot.

In order to analyze CGB, the sheet was divided into 9 lanes of equal width which were numbered from 1 , the left lane, to 9 , the right lane, for convenience. The rate of CGB or \% breakdown time of CGs in each lane was calculated from the data on the sheet. To determine the propagation velocity of CGB, we analyzed the samples in which CGB started from a short side of the rectangle during fertilization. In the case of microinjection, CGB started at the center of the monitor and propagated to its periphery since the microinjection site was in the center of the monitor (see ref. 12).

\section{RESULTS}

Breakdown of single CGs. As summarized in Table I, CGs varied in size and density with species. The distances among adjacent CGs in sand dollar eggs were larger than those in sea urchin eggs. The diameter and the density of CGs in $H$. pulcherrimus and C. japonicus eggs coincided with those reported by Endo who measured them by phase contrast microscopy (4). In $C$. japonicus eggs, some parts of $\mathrm{CG}$ inclusion appeared to be dark, which may be due to the heterogeneity of the inclusion. It is well known by means of electron microscopy that CG inclusion is not homogeneous but heterogeneous (for a review, see ref. 11). In this study, this fact was confirmed in living cells using light microscopy, which may be attributable to the high visibility caused by the facts that the egg cytoplasm is transparent and that CGs were the largest among the four species.

The process of the breakdown of a single $C G$ was analyzed in detail using a high-speed video system at 200 fields/sec. Figure 1 shows the process of CGB in a $C$. japonicus egg upon fertilization. First, the center of the CG became slightly low in contrast, whereas its size did not yet change (see the micrograph of $10 \mathrm{msec}$ in Fig. 1). In 30-40 msec, it swelled up and increased in diameter up to about 1.2 times, and finally disappeared (Fig. 1). In some CGs of the C. japonicus egg, a part of the inclusion remained visible around them after breakdown. Table II summarizes the results of the time required for the breakdown of CGs. The average time was $34 \pm 6$ msec in $H$. pulcherrimus and $32 \pm 8 \mathrm{msec}$ in $C$. japonicus. When CGs in C. japonicus eggs were divided into three groups of small, middle, and large granules in diameter, there was no difference in the average time of CGB among these three groups. These results suggest that the period required for the CGB was almost constant, although the size of CGs varied.

Rate and Propagation of $C G B$. Figure $2 \mathrm{a}$ shows a

Table I. Cortical granules of Sea urchins

\begin{tabular}{lccc}
\hline & Diameter in $\mu \mathrm{m}(\mathrm{n})$ & Density in no. $/ \mu \mathrm{m}^{2}(\mathrm{n})$ & Egg diameter in $\mu \mathrm{m}$ \\
\hline T. hardwicki & $0.62 \pm 0.11(113)$ & $2.34 \pm 0.23(3)$ & $85-95$ \\
H. pulcherrimus & $0.88 \pm 0.20(88)$ & $1.21 \pm 0.06(4)$ & $95-105$ \\
S. mirabilis & $0.71 \pm 0.11(20)$ & $1.05 \pm 0.09(3)$ & $105-110$ \\
C. japonicus & $0.95 \pm 0.21(58)$ & $0.51 \pm 0.02(3)$ & $110-120$ \\
\hline
\end{tabular}

Table II. Periods ReQUired for breakdown of the CORTICAl granule (msec).

\begin{tabular}{lcccc}
\hline & Insemination $(\mathrm{n})$ & $\mathrm{Ca}^{2+}$ injection $(\mathrm{n})$ & Isolated cortex $(\mathrm{n})$ & Total experiments $(\mathrm{n})$ \\
\hline H. pulcherrimus & $33 \pm 7(19)$ & $36 \pm 5(23)$ & - & $34 \pm 6(42)$ \\
C. japonicus & $32 \pm 6(65)$ & $30 \pm 6(41)$ & $34 \pm 11(58)$ & $32 \pm 8(164)$ \\
\hline
\end{tabular}




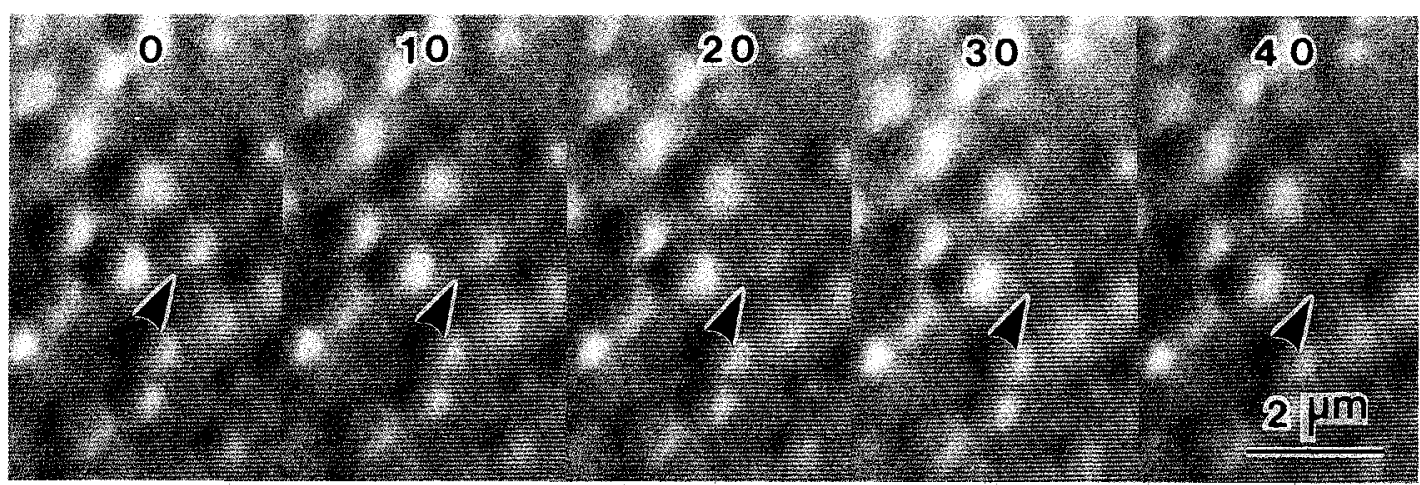

Fig. 1. The process of breakdown of a single CG. A single CGB in a C. japonicus egg upon fertilization was recorded by a high-speed video system at the speed of 200 fields/sec. Numbers in the photograph present the times (msec) from the previous field of the video image where any morphological change such as hollowing of the central area of CG and swelling of CG itself was detected. Arrowhead shows a breaking CG.

scheme which illustrates an egg and the egg surface in the video monitor. CGB propagated from the left side to the right side in the figure during insemination. Figure $2 \mathrm{~b}$ shows the $\%$ breakdown of each lane in $C$. japonicus upon fertilization. In order to calculate the propagation velocity of CGB, times when $10,20,30, \ldots$, and $90 \%$ of total CGs broke down were determined from the data of the number of CGB per second in each lane, and among them, the times of 10,50 , and $90 \%$ breakdown were plotted with the distance in each lane in Fig. 3. Because the slopes of these curves represent the propagation velocities, they were calculated from the times of 10 to $90 \%$ breakdown and were found to be almost the same, namely, $4.0 \mu \mathrm{m} / \mathrm{sec}$ in this sample and
$3.0-4.0 \mu \mathrm{m} / \mathrm{sec}$ in the other samples $(3.3 \pm 0.5 \mu \mathrm{m} / \mathrm{sec}$, $\mathrm{n}=4$ ). Therefore, CGB is considered to occur in the same pattern in each lane except for a time delay to propagate.

During the process of CGB, adjacent CGs do not break down shortly after the propagating stimulus to break down CGs, but seem to break down at random, as mentioned in INTRODUCTION. In order to determine the process of CGB in a small area of the egg surface more precisely, we calculated the mean $\%$ breakdown and its rate per second from the data of 9 lanes after correcting the propagating time of CGB through these lanes so that CGB in all lanes would begin simultaneously, as described below. First, we calculated the a

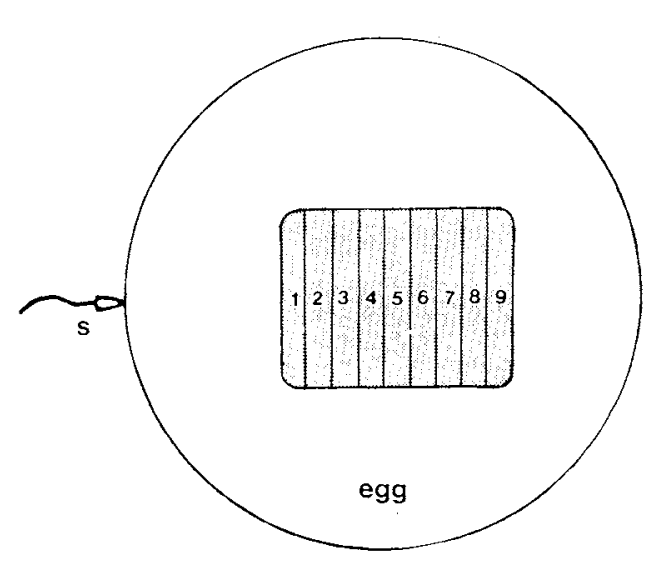

b

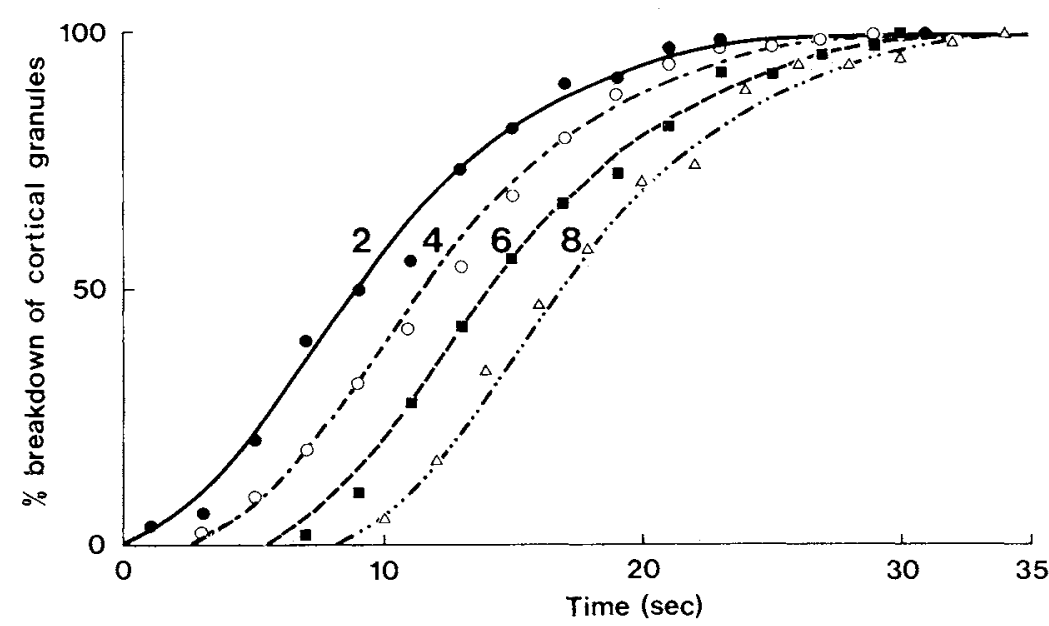

Fig. 2. The breakdown process of CGs in a C. japonicus egg. a is schematic representation of an echinoderm egg during fertilization and of the surface area analyzed with a video monitor. The image field on the video monitor is the real size of the observation area on the egg surface. The parallel lines in the monitor show the divided lines of lanes which are drawn on a transparent plastic sheet in order to determine the propagation velocity of CGB. The numbers in the lanes indicate the lane numbers. $\mathrm{S}$ is fertilizing sperm. $\mathrm{b}$ shows the $\%$ breakdown of CGs in lane 2 , lane 4 , lane 6 , and lane 8 of the image field in a. Numbers represent lane no. 


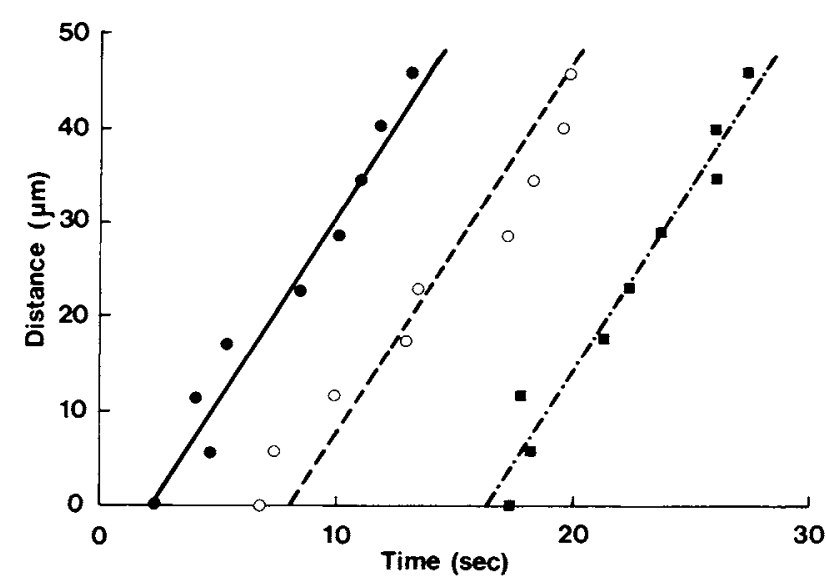

Fig. 3. Propagation of $\%$ breakdown in a C. japonicus egg. The times when $10,20,30, \ldots$, and $90 \%$ of total CGs broke down were determined from the data of the number of CGB per second in each lane. This figure shows the times of 10,50 , and $90 \%$ breakdown in lanes 1-9, which are represented by the distance from lane 1 in the ordinate. The gradients of these lines represent propagation velocities of CGB. The solid line and closed circles show 10\% breakdown; the broken line and open circles, $50 \%$ breakdown; and the chain line and closed squares, $90 \%$ breakdown.

propagating times when CGB propagated from lane 1 to the other lanes from the propagation velocity of CGB and the distances between lane 1 and the other lanes, subtracted these times from the breakdown times of CG in 2-9 lanes, and finally, averaged the \% breakdown of CGs and its rate per second of all lanes. These procedures show in part that the curves in Fig. $2 \mathrm{~b}$ are shifted toward the ordinate and superimposed on the curve of lane 1 . Figure 4 represents the mean $\%$ breakdown of CGs and its rate per second in one sample. The curve of the mean $\%$ breakdown per second has the peak at $7 \mathrm{sec}$, with a shoulder at $13 \mathrm{sec}$. The biphasic tendency was found in all other analyzed samples. These results suggest that CGs consisted of fast and slow breakdown groups according to the responsiveness to the breakdown stimulus. The curve of the mean $\%$ breakdown of CGs shows that it took 8.8 and $17.5 \mathrm{sec}$ for $50 \%$ and $90 \%$, respectively, of CGs to break down. This suggests that when $90 \%$ of CGs in a lane broke down $17.5 \mathrm{sec}$ after the beginning of CGB in the lane, CGs would begin to break down in the surface area $70.0 \mu \mathrm{m}$ distant from the lane because the propagation velocity was $4.0 \mu \mathrm{m} / \mathrm{sec}$ in this sample.

The process of $C G B$ by means of microinjection. The propagation velocity of CGB induced by microinjection of Ca-EGTA buffer or $\mathrm{IP}_{3}$ solution was calculated by the same procedure as in the case of fertilization. However, because the breakdown began in lane 5, the times at each interval of $10 \%$ breakdowns were calculated in 5, and pairs of 4 and 6,3 and 7,2 and 8, and 1 and 9 lanes and plotted with the distance from the center of lane 5 (data not shown). The propagation velocities of CGB induced by microinjection of $\mathrm{Ca}$ EGTA buffer and the $\mathrm{IP}_{3}$ solution were $68 \mu \mathrm{m} / \mathrm{sec}$ and $35 \mu \mathrm{m} / \mathrm{sec}$, respectively, when calculated by $50 \%$ breakdown times.

$C G B$ does not affect the breakdown of neighboring CGs. To analyze quantitatively how the breakdown of a CG would affect the breakdown of neighboring CGs around the $C G$, we randomly took two pairs of CGs in directions both parallel and perpendicular to the

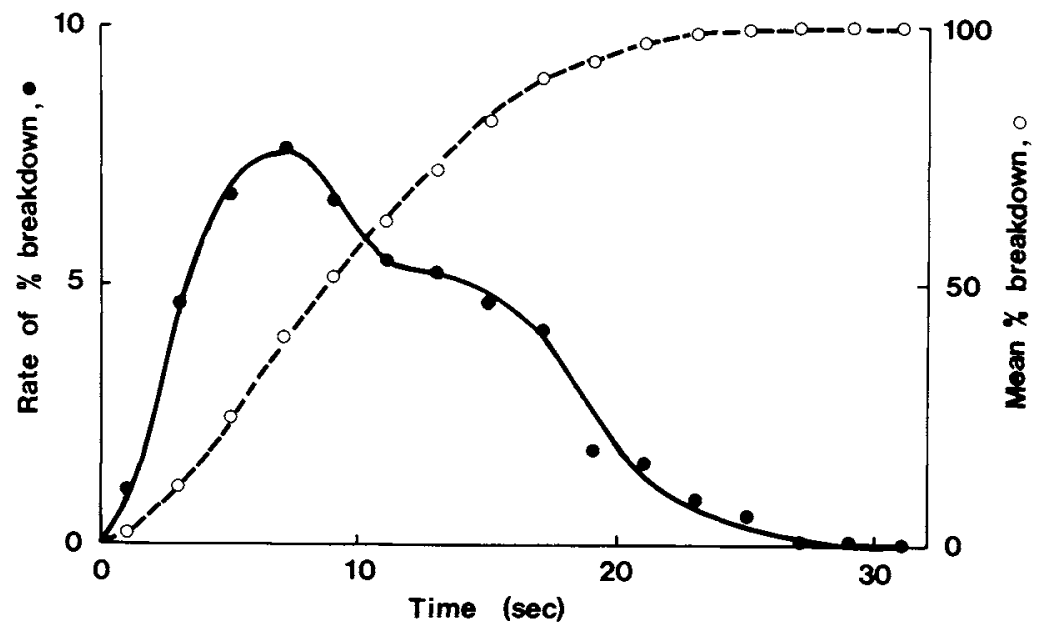

Fig. 4. The mean \% breakdown of CGs and the rate of the mean \% breakdown of CGs versus time in a C. japonicus egg. When assuming that the beginning time of CGB in each lane was the same by correcting each propagating time, the mean $\%$ breakdown of CGs and the rate of mean $\%$ breakdown of CGs were determined. It is noted that there is a shoulder on the curve of the rate of mean $\%$ breakdown after the peak. Closed circles show the rate of mean $\%$ breakdown and open circles, the mean $\%$ breakdown. 
Table III. Differences between bREakdown times of two cortical granules.

\begin{tabular}{|c|c|c|c|c|}
\hline Sample & $\begin{array}{l}\text { Short distance } \\
\text { in } \mu \mathrm{m}\end{array}$ & $\begin{array}{c}\text { Time difference in sec } \\
\text { in the case of short distance (n) }\end{array}$ & $\begin{array}{l}\text { Long distance } \\
\text { in } \mu \mathrm{m}\end{array}$ & $\begin{array}{l}\text { Time difference in sec } \\
\text { in the case of long distance (n) }\end{array}$ \\
\hline \multicolumn{5}{|c|}{ Fertilization } \\
\hline \multirow[t]{2}{*}{1} & $\mathrm{dx} \quad 1.7 \pm 0.2$ & $0.2 \pm 6.6(31)$ & $21.3 \pm 0.5$ & $8.0 \pm 9.4(30)$ \\
\hline & dy $1.8 \pm 0.2$ & $-0.9 \pm 6.1(31)$ & $21.4 \pm 0.5$ & $-0.6 \pm 9.5(30)$ \\
\hline \multirow[t]{2}{*}{2} & $\mathrm{dx} \quad 1.8 \pm 0.2$ & $0.7 \pm 6.8(30)$ & $21.3 \pm 0.5$ & $6.8 \pm 6.3(30)$ \\
\hline & dy $\quad 1.8 \pm 0.2$ & $1.3 \pm 7.7(30)$ & $21.3 \pm 0.4$ & $-1.2 \pm 7.8(30)$ \\
\hline \multirow[t]{3}{*}{3} & $\mathrm{dx} \quad 1.7 \pm 0.2$ & $1.5 \pm 6.6(30)$ & $21.3 \pm 0.5$ & $4.8 \pm 5.6(30)$ \\
\hline & dy $\quad 1.8 \pm 0.2$ & $0.7 \pm 8.2(30)$ & $21.3 \pm 0.4$ & $1.9 \pm 7.0(30)$ \\
\hline & \multicolumn{4}{|c|}{ Microinjection } \\
\hline C-E & $\mathrm{dr} \quad 1.9 \pm 0.3$ & $-0.3 \pm 1.3(30)$ & $15.5 \pm 0.7$ & $0.3 \pm 1.2(30)$ \\
\hline $\mathrm{IP}_{3}$ & $\mathrm{dr} \quad 2.1 \pm 0.3$ & $0.1 \pm 1.2(30)$ & $15.9 \pm 0.7$ & $0.7 \pm 1.6(30)$ \\
\hline
\end{tabular}

Time difference indicates the time from one breakdown to the other of a pair of CGs which are taken randomly in the observation area. dx: distance between a pair of CGs to the direction parallel to the propagation. dy: distance between a pair of CGs to the direction perpendicular to the propagation. dr: distance between a pair of CGs to the radial direction from the microinjection site. C-E: Ca-EGTA buffer.

direction of propagation in the case of fertilization or a pair of CGs in the direction of propagation in the case of microinjection, as shown in Table III. The distance between the pair was short (about $2 \mu \mathrm{m}$ ) or long (ca. 21 or $16 \mu \mathrm{m}$ ). If the breakdown of a CG accelerates or decelerates the breakdown of neighboring CGs, the breakdown time of CG depends on the distance, or if CGB has no relation to the breakdown of neighboring CGs, the breakdown time of $\mathrm{CG}$ does not depend on the distance. Differences between breakdown times of a pair of CGs are summarized in Table III. Standard deviations themselves show the degree of randomness. In this study, they were large and did not depend on the direction or the distance between the pair of CGs. These results indicate that CGB does not accelerate or decelerate the breakdown of neighboring CGs, but that CGs break down independently of the breakdown of neighboring CGs. The mean shows the time when a CGB stimulus propagates to the other CGB at a definite distance. Although the propagation velocity of CGB could also be calculated by this mean and should be equal to the values described in the two previous subsections, it could not be determined exactly because the deviation was large compared to the mean.

\section{DISCUSSION}

Process of single CGB. The process of single CGB was investigated using a high-speed video system at a speed of 200 fields/sec. It was found that a CG swelled up by approximately $20 \%$ in diameter and then broke down. Our data did not coincide with the findings of Endo that a CG swelled in diameter by a factor of two during CGB (4). Since we observed the phenomenon that CG inclusions swelled up significantly after CGB, it is suggested that there was confusion between the swelling of a CG before the breakdown and the swelling of
CG inclusions after the breakdown. The time of fusion pore opening could not be determined in this study. However, it seems likely that a CG would open first after the fusion of the $\mathrm{CG}$ and egg plasma membrane and that the CG swells up, increasing the size of the opening pore because it has been reported that a secretory vesicle in beige mice swelled up after the fusion of the vesicle and cell membrane $(2,3)$.

Propagation of CGB. CGB starts at the sperm attachment site and spreads over the entire surface of the egg $(10,11,19,23)$. In C. japonicus, the propagation velocity of CGB was in the range of $3.0-4.0 \mu \mathrm{m} / \mathrm{sec}$, which coincides with those of cortical changes reported as $3-4 \mu \mathrm{m} / \mathrm{sec}(9,16,24)$. However, the velocities were large in some reports $(4,13)$. Upon fertilization, the velocity of the Ca-wave in echinoderm eggs was measured to be $3-4 \mu \mathrm{m} / \mathrm{sec}$ using the fluorescent dye, fura-2 (in preparation). A transient $\mathrm{Ca}^{2+}$ increase was also reported to propagate through the egg cytoplasm $(5,6$, 22). Therefore, CGB may propagate at the same velocity of a transient $\mathrm{Ca}^{2+}$ increase upon fertilization. Moreover, we reported that the first CGB occurred about 0.5 sec after increasing $\mathrm{Ca}^{2+}$ concentration in the cytoplasm by microinjection (12), and the fertilization envelope was reported to elevate $10.5 \mathrm{sec}$ after the beginning of $\mathrm{Ca}^{2+}$ increase upon fertilization (6). These data indicate that the stimulus to induce CGB is intracellular $\mathrm{Ca}^{2+}$ increase, that the first $\mathrm{CGB}$ would be detected at about $0.5 \mathrm{sec}$ of a latent period after the $\mathrm{Ca}^{2+}$ increase and that $10 \mathrm{sec}$ later when $60 \% \mathrm{CGs}$ broke down, the fertilization envelope elevation would be detected. The propagation velocities of CGB induced by Ca-EGTA buffer and $\mathrm{IP}_{3}$ solutions were about 20 times and 10 times as much as that upon fertilization, respectively. This may be because Ca-EGTA and EGTA in CaEGTA buffer, and $\mathrm{IP}_{3}$ diffused much faster in the egg cytoplasm than the propagation of $\mathrm{Ca}^{2+}$ increase 
during fertilization and because the velocities were influenced by injectates which diffused directly from the injection site.

Relationship between CGBs. $\mathrm{Ca}^{2+}$ increase is thought to be a stimulus to induce CGB. Nevertheless, CGB was not simply induced by $\mathrm{Ca}^{2+}$ increase because all adjacent CGs did not break down simultaneously after $\mathrm{Ca}^{2+}$ increase but broke down gradually during more than $20 \mathrm{sec}$. Therefore, the relationship between CGBs was analyzed by taking two pairs of CGs with short and long distances in this study and it was found that CGs seemed to break down at random. However, this randomness may not be due to the fluctuation of concentration of $\mathrm{Ca}^{2+}$ in the egg cytoplasm near a CG, as described below. It has been reported that the peak concentration of $\mathrm{Ca}^{2+}$ in the sea urchin egg was a few $\mu \mathrm{M}(5,15,21,22)$. Since $\mathrm{Ca}^{2+}$ concentration in $\mathrm{Ca}-$ EGTA buffer in the case of our microinjection experiment was $6 \mu \mathrm{M}$, the number of $\mathrm{Ca}^{2+}$ molecules is about $3600 / \mu \mathrm{m}^{3}$. On the other hand, if the concentration of $\mathrm{Ca}^{2+}$ in the egg cytoplasm fluctuates at $6 \mu \mathrm{M}$ during fertilization and if the fluctuation of $\mathrm{Ca}^{2+}$ depends on the Poisson process, the fluctuation of $\mathrm{Ca}^{2+}$ is calculated to be only $60 / \mu \mathrm{m}^{3}$ and less than $2 \%$ of $3600 / \mu \mathrm{m}^{3}$. Moreover, if the fluctuation is caused by Brownian motion, the displacement $(\mathrm{dx})$ of $\mathrm{Ca}^{2+}$ is expressed by the equation as follows:

$$
\left.<\mathrm{dx}^{2}\right\rangle=2 \mathrm{Dt},
$$

where $\mathrm{D}$ is a diffusion constant and $\mathrm{t}$ is time, using the Einstein-Stokes relation. Provided $\mathrm{D}=1.0 \times 10^{-10} \mathrm{~m}^{2} /$ sec from the result of Hiramoto and Kaneda's study (8) in C. japonicus, it can be determined that it takes 5 msec for $\mathrm{Ca}^{2+}$ to move about $1 \mu \mathrm{m}$, which is equal to the diameter of CGs. This indicates that $\mathrm{Ca}^{2+}$ near a CG diffuses in the surrounding cytoplasm within such a very short time such as $5 \mathrm{msec}$, whereas CGB occurs for more than $20 \mathrm{sec}$ in a definite surface area. Consequently, the fluctuation of $\mathrm{Ca}^{2+}$ concentration in the egg cytoplasm cannot explain the randomness of CGB. Therefore, CGB may well involve other processes of probability constituting some biological mechanism other than the $\mathrm{Ca}^{2+}$ increase itself. Some candidates for the process may be enzymatic reactions involving calmodulin and other $\mathrm{Ca}^{2+}$ binding proteins $(14,17,20)$.

Heterogeneity of CGs. It is intriguing that the mean $\%$ breakdown of CGs per second is biphasic, as shown in Fig. 4. There may be heterogeneity in a population of CGs with respect to the reactivity of CGB. Differences between the two populations may be characterized only by the differences in the reaction time of CGB to a stimulus because the average period required for CGB was almost constant (approximately $40 \mathrm{msec}$ ) and the propagation velocities of CGB were also constant (about $3.3 \mu \mathrm{m} / \mathrm{sec}$ ). However, each population may in- volve other biological functions, since it was reported that an immunocytochemical technique suggested heterogeneity in a population of sea urchin egg CGs (1).

Acknowledgments. We wish to thank Emeritus Prof. Yukio Hiramoto of Tokyo Institute of Technology for his valuable advice and critical reading of the manuscript. We also wish to thank the staff of Ushimado Marine Biological Station and Misaki Marine Biological Station for supplying the materials, and wish to thank Nac Inc. for the generous loan of the Nac microscopic high-speed video.

This work was supported by a Grant-in-Aid for Scientific Research from the Japan Ministry of Education, Science, and Culture to Y. H. (No. 62540538).

\section{REFERENCES}

1. Anstrom, J.A., Chin, J.E., Leaf, D.S., Parks, A.L., and RAFF, R.A. (1988). Immunocytochemical evidence suggesting heterogeneity in the population of sea urchin egg cortical granules. Dev. Biol., 125: 1-7.

2. Breckenridge, L.J. and Almers, W. (1987). Final steps in exocytosis observed in a cell with giant secretory granules. Proc. Natl. Acad. Sci. USA, 84: 1945-1949.

3. BreCKenRidge, L.J. and Almers, W. (1987). Currents through the fusion pore that forms during exocytosis of secretory vesicle. Nature, 328: 814-817.

4. Endo, Y. (1952). The role of cortical granules in the formation of the fertilization membrane in eggs from Japanese sea urchin. I. Exp. Cell Res., 3: 406-418.

5. Hafner, M., Petzelt, C., Nobiling, R., Pawley, J.B., Kramp, D., and Schatten, G. (1988). Wave of free calcium at fertilization in the sea urchin egg visualized with fura-2. Cell Motil. Cytoskel., 9: 271-277.

6. Hamaguchi, Y. and Hamaguchi, M.S. (1990). Simultaneous investigation of intracellular $\mathrm{Ca}^{2+}$ increase and morphological events upon fertilization in the sand dollar eggs. Cell Struct. Funct., 15: 159-162.

7. Hiramoto, Y. (1955). Nature of the perivitelline space in sea urchin eggs. III. On the mechanism of membrane elevation. Annot. Zool. Japon., 28: 183-193.

8. Hiramoto, Y. and Kaneda, I. (1988). Diffusion of substances in the cytoplasm and across the nuclear envelope in egg cells. Protoplasma, supple., 2: 183-193.

9. KACSER, H. (1955). The cortical changes on fertilization of the sea-urchin egg. J. Exp. Biol., 32: 451-467.

10. KAY, E.R. and SHAPIRO, B.M. (1985). The formation of fertilization membrane of sea urchin egg. In Biology of Fertilization vol. 3 (C.B. Metz, and A. Monroy, eds.). Academic Press, London, pp45-73.

11. Longo, F.J. (1989). Egg cortical architecture. In The Cell Biology of Fertilization (H. Schatten, and G. Schatten, eds.). Academic Press, Inc., pp105-138.

12. Mohri, T. and Hamaguchi, Y. (1989). Analysis of the breakdown of cortical granules in echinoderm eggs by microinjection of second messengers. Cell Struct. Funct., 14: 429-438.

13. Moser, F. (1939). Studies on a cortical layer response to stimulating agents in the Arbacia egg. I. Response to insemination. $J$. Exp. Zool., 80: 423-471.

14. PiCard, A. and Doree, M. (1982). Intracellular microinjection of anticalmodulin drugs does not inhibit the cortical reaction induced by fertilization, ionophore A 23187 or injected calcium buffers in sea urchin eggs. Develop. Growth. and Differ., 
24: $155-162$.

15. Poenie, M., Alderton, J., Tsien, R.Y., and Steinhardt, R.A. (1985). Changes of the free calcium levels with stages of the cell division cycle. Nature, 315: 147-149.

16. RothschILD, L. and Swann, M.M. (1949). The fertilization reaction in the sea urchin egg. J. Exp. Biol., 26: 164-176.

17. SASAKI, H. (1984). Modulation of calcium sensitivity by a specific cortical protein during sea urchin egg cortical vesicle exocytosis. Dev. Biol., 101: 125-135.

18. SASAKI, H. and Epel, D. (1983). Cortical vesicle exocytosis in isolated cortices of sea urchin eggs: Description of a turbidometric assay and its utilization in studying effect of different media on discharge. Dev. Biol., 98: 327-337.

19. Schuel, H. (1985). Function of egg cortical granules. In Biology of fertilization, vol. 3 (C.B. Metz, and A. Monroy, eds.). Academic Press, London, pp1-43.
20. Steinhardt, R.A. and Alderton, J.M. (1982). Calmodulin confers calcium sensitivity on secretory exocytosis. Nature, 295: 154-155.

21. Steinhardt, R.A., Zucker, R., and Schatten, G. (1977). Intracellular calcium release at fertilization in the sea urchin egg. Dev. Biol., 58: 185-196.

22. SWAnn, K. and Whitaker, M. (1986). The part played by inositol trisphosphate and calcium in the propagation of the fertilization wave in sea urchin eggs. J. Cell. Biol., 103: 2333-2342.

23. TRIMMER, J.S., and VACQUIER, V.D. (1986). Activation of sea urchin gametes. Ann. Rev. Cell Biol., 2: 1-26.

24. WhitaKer, M.J. and Steinhardt, R.A. (1985). Ionic signaling in the sea urchin egg at fertilization. In Biology of fertilization, vol. 3 (C.B. Metz, and A. Monroy, eds.). Academic Press, London, pp167-221.

(Received for publication, July 3, 1990

and in revised form, August 9, 1990) 